PROCEEDINGS OF THE

AMERICAN MATHEMATICAL SOCIETY

Volume 130, Number 3, Pages 689-696

S 0002-9939(01)06225-6

Article electronically published on July 25, 2001

\title{
SOLUTIONS TO A CLASS OF SCHRÖDINGER EQUATIONS
}

\author{
YANHENG DING
}

(Communicated by David S. Tartakoff)

\begin{abstract}
We establish existence and multiplicity of solutions to a class of nonlinear Schrödinger equations with, e.g., "atomic" Hamiltonians, via critical point theory.
\end{abstract}

\section{INTRODUCTION AND RESULTS}

Consider the Schrödinger equation

$$
-i \frac{d \varphi}{d t}=A \varphi+G_{\varphi}(x, \varphi)
$$

where $A$ stands for a Schrödinger operator in $L^{2}\left(\mathbb{R}^{3}\right)$, and $G: \mathbb{R}^{3} \times \mathbb{C} \rightarrow \mathbb{R}$ a nonlinear coupling with $G(x, 0)=0$ and $G\left(x, e^{i \theta t} u\right)=G(x, u)$. We are interested in existence and multiplicity of solutions of the form

$$
\varphi(t, x)=e^{i \lambda t} u(x) \quad \text { with } \quad u \in H^{1}\left(\mathbb{R}^{3}\right) .
$$

Substituting it in (1.1) one sees that $u$ satisfies

$$
\left\{\begin{array}{l}
A u-\lambda u+g(x, u)=0 \\
u \in H^{1}\left(\mathbb{R}^{3}\right)
\end{array}\right.
$$

where $g(x, u):=G_{u}(x, u)$. In this paper we deal with the following Schrödinger operators:

- The Schrödinger operator with Rollnik potentials

$$
A_{1}:=-\Delta+V(x),
$$

that is, for any $\epsilon>0$, there is an expression $V=V_{1}+V_{2} \in \mathcal{R}+\left(L^{\infty}\right)_{\epsilon}$ where $\mathcal{R}$ is the class of real functions $W$ satisfying

$$
\|W\|_{\mathcal{R}}^{2}:=\int_{\mathbb{R}^{3} \times \mathbb{R}^{3}} \frac{|W(x)||W(y)|}{|x-y|^{2}} d x d y<\infty,
$$

and $\left(L^{\infty}\right)_{\epsilon}$ indicates the class of real functions $W$ satisfying $\|W\|_{\infty}<\epsilon$. Suppose that $V(x) \leq-a r^{-2+\epsilon}$ for $r:=|x|>R_{0}$ some $R_{0}$ and $a>0, \epsilon>0$.

Received by the editors August 15, 2000.

1991 Mathematics Subject Classification. Primary 35Q55; Secondary 58E55.

Key words and phrases. Schrödinger equations, multiple solutions, critical point theory.

This research was supported by the Special Funds for Major State Basic Research Projects of China.

(C)2001 American Mathematical Society 
- The Hamiltonian of the helium atom

$$
A_{2}:=-\Delta_{1}-\Delta_{2}-\frac{2}{\left|\mathbf{r}_{1}\right|}-\frac{2}{\left|\mathbf{r}_{2}\right|}+\frac{1}{\left|\mathbf{r}_{1}-\mathbf{r}_{2}\right|}
$$

where $\mathbf{r}_{i}=\left(x_{1}, x_{2}, x_{3}\right) \in \mathbb{R}^{3}$ and $\Delta_{i}=\sum_{k=1}^{3} \frac{\partial^{2}}{\partial x_{k}^{2}}$.

- The general "atomic" Hamiltonian of the form

$$
A_{3}:=\sum_{i=1}^{m}\left(-\frac{\Delta_{i}}{2 \mu_{i}}-\frac{m}{\left|\mathbf{r}_{i}\right|}\right)+\sum_{i<j}\left(\frac{\nabla_{i} \cdot \nabla_{j}}{M}+\frac{1}{\left|\mathbf{r}_{i}-\mathbf{r}_{j}\right|}\right),
$$

a system consisting of the nucleus of mass $M$ and $m$ electrons of masses $\mu_{1}, \cdots, \mu_{m}$ after the center of mass motion is removed.

Recall that the operator $A=A_{i}(i=1,2,3)$ is selfadjoint with $\mathcal{D}\left(A_{i}\right) \subseteq H^{2}\left(\mathbb{R}^{3}\right)$. Moreover, the bound state energies $\sigma\left(A_{i}\right) \cap\left[\lambda_{0}, \lambda_{e}\right)$ is an infinite set (cf. [13]), where $\lambda_{0}:=\inf \sigma(A), \lambda_{e}:=\inf \sigma_{\text {ess }}(A) \quad\left(\sigma(A)\right.$ and $\sigma_{\text {ess }}(A)$ denote the spectrum and essential spectrum). Assume first that $g$ satisfies the following superlinear hypotheses:

$(g 1) g(x, u)=o(|u|)$ uniformly with respect to $x$ as $u \rightarrow 0$;

(g2) there exist $\gamma>2, \delta>0$ such that $0<\gamma G(x, u) \leq u g(x, u)$ whenever $u \neq 0$ and $G(x, u) \geq \delta$ whenever $|u|=1$;

(g3) there are $p>2$ and $c>0$ such that

$$
|g(x, u+v)-g(x, u)| \leq c\left(1+|u|^{p-2}+|v|^{p-2}\right)|v| .
$$

For obtaining multiple solutions we assume $g$ is odd in $u$, that is,

(g4) $g(x,-u)=-g(x, u)$.

In the following, for any $\lambda \leq \lambda_{e}$, let $\mathcal{N}(\lambda)$ denote the number of all eigenvalues of $A$ which are less than $\lambda$ and counted in multiplicity.

Theorem 1. ( $i)$ Assume $(g 1)-(g 3)$. Then $(\mathrm{S})$ has no nontrivial solutions if $\lambda \leq$ $\lambda_{0}$, and has at least two nontrivial solutions if $\lambda \in\left(\lambda_{0}, \lambda_{e}\right)$.

(ii) Assume $(g 1)-(g 3)$ and $(g 4)$. Then $(\mathrm{S})$ has at least $\mathcal{N}(\lambda)$ pairs of solutions.

Next consider the case where $g(x, u)$ is sublinear with respect to $u \in \mathbb{R}$. Assume (g5) $g(x, u) u \geq 0$, and there are $1<\alpha \leq \beta<2, c_{1}, c_{2}>0$ such that $|g(x, u)| \leq$ $c_{1}\left(|u|^{\alpha-1}+|u|^{\beta-1}\right)$ and $2 G(x, u)-g(x, u) u \geq c_{2}|u|^{\alpha}$.

Theorem 2. ( $i)$ Assume (g5) holds. Then (S) has no nontrivial solutions if $\lambda \leq$ $\lambda_{0}$, and has at least one nontrivial solution if $\lambda \in\left(\lambda_{0}, \lambda_{e}\right)$.

(ii) Assume $(g 4)-(g 5)$ hold. Then $(\mathrm{S})$ has $\mathcal{N}(\lambda)$ pairs of solutions.

A typical example satisfying the above hypotheses is $g(x, u)=|u|^{p-2} u$. With this nonlinearity, the above results conclude that the problem $(\mathrm{S})$ has $\mathcal{N}(\lambda)$ pairs of solutions provided $p \in(1,2) \cup(2, \infty)$ and $\lambda \in\left(\lambda_{0}, \lambda_{e}\right)$. Clearly, if $p=2$, (S) has solutions if and only if $\lambda-1$ is an eigenvalue of $A$.

The problems looking for stationary solutions to Schrödinger equations have been studied extensively via variational methods. We refer, e.g., to [10] for HartreeFock equations, [5] for Schrödinger operators of atoms and molecules, and [1], 3], [4], [6], [8], [12], [14] for the Schödinger operators with continuous (periodic etc.) potentials. We also mention that a similar problem of semilinear elliptic equations on bounded domains was studied in [2], [7]. The arguments used below extend to higher dimensions and more operators. 


\section{THE SUPERLINEAR CASE}

Fix $\lambda<\lambda_{e}$ and set $A_{\lambda}:=A-\lambda$. Let $E^{+}$be the subspace of $L^{2}\left(\mathbb{R}^{3}\right)$ spanned by all eigenfunctions corresponding to negative eigenvalues of $A_{\lambda}$, and let $E^{0}:=\operatorname{ker} A_{\lambda}$. Then $\operatorname{dim} E^{+}=\mathcal{N}(\lambda)$ and $L^{2}=E^{+} \oplus E^{0} \oplus L^{-}, u=u^{+}+u^{0}+u^{-}$, where $L^{-}$ denotes the orthogonal complement of $E^{+} \oplus E^{0}$ in $L^{2}$. Let $E:=\mathcal{D}\left(\left|A_{\lambda}\right|^{1 / 2}\right)$ be the Hilbert space with the inner product

$$
(u, v):=\left(\left|A_{\lambda}\right|^{1 / 2} u,\left|A_{\lambda}\right|^{1 / 2} v\right)_{L^{2}}+\left(u^{0}, v^{0}\right)_{L^{2}}
$$

and norm $\|u\|=(u, u)^{1 / 2}$, where $(\cdot, \cdot)_{L^{2}}$ denotes the usual $L^{2}$-inner product. There is a decomposition

$$
E=E^{+} \oplus E^{0} \oplus E^{-}, \quad u=u^{+}+u^{0}+u^{-}
$$

with $E^{-}=L^{-} \cap E$, orthogonal with respect to both $(\cdot, \cdot)_{L^{2}}$ and $(\cdot, \cdot)$. Since $\mathcal{D}(A) \subseteq$ $H^{2}\left(\mathbb{R}^{3}\right), E$ is embedded continuously in $H^{1}\left(\mathbb{R}^{3}\right)$, hence continuously in $L^{t}\left(\mathbb{R}^{3}\right)$ for all $t \in[2,6]$ and compactly in $L_{l o c}^{t}\left(\mathbb{R}^{3}\right)$ for all $t \in[1,6)$.

Now, let $E_{p}$ be the Banach space of the completion of $\mathcal{C}_{0}^{\infty}\left(\mathbb{R}^{3}\right)$ in the norm $\|u\|_{p}=\left(\|u\|^{2}+|u|_{p}^{2}\right)^{1 / 2}$. Here and below by $|\cdot|_{p}$ we denote the usual $L^{p}$-norm. By the Sobolev embedding theorem $E_{p}=E$ if $p \in[2,6]$. In general, $E_{p}$ embeds continuously in $L^{s}$ for all $s \in[p, 6]$ (resp. $s \in[2, p]$ ) if $p \in(1,6)$ (resp. $p \geq 6$ ). It is clear that $E_{p}$ possesses the direct sum decomposition

$$
E_{p}=E^{+} \oplus E^{0} \oplus E_{p}^{-}, \quad u=u^{+}+u^{0}+u^{-}
$$

where $E_{p}^{-}$is the completion of $\mathcal{C}_{0}^{\infty}\left(\mathbb{R}^{3}\right) \cap L^{-}$under the norm $\|\cdot\|_{p}$. Throughout this section we assume $(g 1)-(g 3)$ and fix $E_{p}$ with $p>2$ from $(g 3)$. Define

$$
I(u)=I_{\lambda}(u):=\frac{1}{2}\left(\left\|u^{+}\right\|^{2}-\left\|u^{-}\right\|^{2}\right)-\int_{\mathbb{R}^{3}} G(x, u) .
$$

Under the assumptions, $I \in \mathcal{C}^{1}\left(E_{p}, \mathbb{R}\right)$ and solutions of $(\mathrm{S})$ can be found as critical points of $I$. It follows from the assumptions that, for any $\varepsilon>0$, there are $C_{\varepsilon}, C_{\varepsilon}^{\prime}>0$ such that

$$
C_{\varepsilon}|u|^{\gamma}-\varepsilon|u|^{2} \leq G(x, u) \leq \varepsilon|u|^{2}+C_{\varepsilon}^{\prime}|u|^{p} \quad \text { for all }(x, u) .
$$

Since $\operatorname{dim}\left(E^{+} \oplus E^{0}\right)<\infty$ and $\gamma>2$, the following lemma clearly holds.

Lemma 2.1. $I$ is bounded from above: $\sup I(E)<\infty$. In addition, there is $R>0$ such that $\sup I\left(E_{p} \backslash B_{R}\right) \leq 0$.

Using (2.1) one gets easily the following

Lemma 2.2. If $\lambda>\lambda_{0}$, there is $\rho>0$ such that $\mu:=\inf I\left(S_{\rho}^{+}\right)>0$ where $S_{\rho}^{+}:=\partial B_{\rho} \cap E^{+}$.

Recall that a sequence $\left(u_{n}\right) \subset E_{p}$ is called a $(\mathrm{PS})_{c}$ sequence if $I\left(u_{n}\right) \rightarrow c$ and $I^{\prime}\left(u_{n}\right) \rightarrow 0 . I$ is said to satisfy the $(\mathrm{PS})_{c}$ condition if any $(\mathrm{PS})_{c}$ sequence has a convergent subsequence.

Lemma 2.3. I satisfies the $(\mathrm{PS})_{c}$ condition.

Proof. Let $I\left(u_{n}\right) \rightarrow c$ and $I^{\prime}\left(u_{n}\right) \rightarrow 0$. A standard argument shows that $\left(u_{n}\right)$ is bounded (cf. 8] or 4, [15]). We can suppose, without loss of generality, that $u_{n} \rightarrow u$. Since $\operatorname{dim}\left(E^{+} \oplus E^{0}\right)<\infty, u_{n}^{0}+u_{n}^{+} \rightarrow u^{0}+u^{+}$. It remains to show that $u_{n}^{-} \rightarrow u^{-}$. 
Set $w_{n}=u_{n}-u$. Then $w_{n} \rightarrow 0$ and $w_{n}(x) \rightarrow 0$ a.e. in $x$ since $E_{p}$ embeds compactly in $L_{l o c}^{2}$. Observe that

$$
\left(I^{\prime}\left(u_{n}\right)-I^{\prime}(u)\right) w_{n}=\left\|w_{n}^{+}\right\|^{2}-\left\|w_{n}^{-}\right\|^{2}-\int_{\mathbb{R}^{3}}\left(g\left(x, u_{n}\right)-g(x, u)\right) w_{n},
$$

or

$$
\left\|w_{n}^{-}\right\|^{2}+\int_{\mathbb{R}^{3}}\left(g\left(x, u_{n}\right)-g(x, u)\right) w_{n}=o(1) .
$$

Write

$$
\begin{aligned}
\int_{\mathbb{R}^{3}}\left(g\left(x, u_{n}\right)-g(x, u)\right) w_{n}= & \int_{\mathbb{R}^{3}} g\left(x, w_{n}\right) w_{n} \\
& +\int_{\mathbb{R}^{3}}\left(g\left(x, w_{n}+u\right)-g\left(x, w_{n}\right)-g(x, u)\right) w_{n} .
\end{aligned}
$$

Fix $\varepsilon>0$. By assumptions it is easy to verify that there is $c_{\varepsilon}>0$ satisfying

$$
\left|g\left(x, w_{n}+u\right)-g\left(x, w_{n}\right)-g(x, u)\right| \leq \varepsilon\left|w_{n}\right|^{p-1}+c_{\varepsilon}\left(|u|+|u|^{p-1}\right) .
$$

Set $\Omega:=\left\{x \in \mathbb{R}^{3}:|u(x)|<1\right\}, \Omega^{c}:=\mathbb{R}^{3} \backslash \Omega$, and

$$
f_{n}^{\varepsilon}:=\left(\left|g\left(x, w_{n}+u\right)-g\left(x, w_{n}\right)-g(x, u)\right|-\varepsilon\left|w_{n}\right|^{p-1}\right)^{+},
$$

where $(a)^{+}:=\max \{a, 0\}$. Then $f_{n}^{\varepsilon} \leq 2 c_{\varepsilon}|u|$ on $\Omega$ and $f_{n}^{\varepsilon} \leq 2 c_{\varepsilon}|u|^{p-1}$ on $\Omega^{c}$. The Lebesgue theorem then implies $\int_{\Omega}\left(f_{n}^{\varepsilon}\right)^{2} \rightarrow 0$ and $\int_{\Omega^{c}}\left(f_{n}^{\varepsilon}\right)^{p^{\prime}} \rightarrow 0$ where $p^{\prime}=p /(p-1)$. Therefore,

$$
\begin{aligned}
& \limsup _{n \rightarrow \infty} \int_{\Omega}\left|g\left(x, w_{n}+u\right)-g\left(x, w_{n}\right)-g(x, u)\right|\left|w_{n}\right| \\
& \leq \limsup _{n \rightarrow \infty}\left(\left(\int_{\Omega}\left(f_{n}^{\varepsilon}\right)^{2}\right)^{1 / 2}\left|w_{n}\right|_{2}+\varepsilon\left|w_{n}\right|_{p}^{p}\right) \leq c \varepsilon
\end{aligned}
$$

and

$$
\begin{aligned}
& \limsup _{n \rightarrow \infty} \int_{\Omega^{c}}\left|g\left(x, w_{n}+u\right)-g\left(x, w_{n}\right)-g(x, u)\right|\left|w_{n}\right| \\
& \leq \limsup _{n \rightarrow \infty}\left(\left(\int_{\Omega^{c}}\left(f_{n}^{\varepsilon}\right)^{p^{\prime}}\right)^{1 / p^{\prime}}\left|w_{n}\right|_{p}+\varepsilon\left|w_{n}\right|_{p}^{p}\right) \leq c \varepsilon
\end{aligned}
$$

where $c:=\sup _{n}\left|w_{n}\right|_{p}^{p}$. We obtain

$$
\int_{\mathbb{R}^{3}}\left(g\left(x, u_{n}\right)-g(x, u)\right) w_{n}=\int_{\mathbb{R}^{3}} g\left(x, w_{n}\right) w_{n}+o(1) .
$$

Substituting in (2.2) yields

$$
\left\|w_{n}^{-}\right\|^{2}+\int_{\mathbb{R}^{3}} g\left(x, w_{n}\right) w_{n}=o(1) .
$$

Since $g\left(x, w_{n}\right) w_{n} \geq 0$, it follows that $u_{n}^{-} \rightarrow u^{-}$.

Now we are ready to prove Theorem 1.

Proof of Theorem 1. ( $i)$ Assume $(g 1),(g 2)$ and $(g 3)$ hold. Remark that if $u$ is a nontrivial critical point of $I$, then

$$
I(u)=I(u)-\frac{1}{2} I^{\prime}(u) u \geq \frac{\gamma-2}{2} \int_{\mathbb{R}^{N}} G(x, u)>0 .
$$


If $\lambda \leq \lambda_{0}$, then $\sup I(E) \leq 0$, so $I$ has no nontrivial critical points by (2.3).

Let $\lambda \in\left(\lambda_{0}, \lambda_{e}\right)$. By Lemma 2.1, $I$ is bounded from above. In virtue of Lemma $2.3 I$ satisfies the $(\mathrm{PS})_{c}$. In addition, it follows from (2.1) that there exist $r, \varepsilon>0$ such that

$$
\sup I\left(\partial B_{r} \cap\left(E^{-} \oplus E^{0}\right)\right) \leq-\varepsilon<\sup I\left(E^{-} \oplus E^{0}\right)=0
$$

and

$$
0=\inf I\left(B_{r} \cap E^{+}\right)<\varepsilon \leq \inf I\left(\partial B_{r} \cap E^{+}\right) .
$$

Therefore $I$ has at least two nontrivial critical points via the well-known "three solution theorem" (cf. [9]).

(ii) Assume ( $g 4)$ also holds. Recall that $I$ is even and bounded from above, satisfies the $(\mathrm{PS})_{c}, I(0)=0$, and $\inf I\left(\partial B_{\rho} \cap E^{+}\right) \geq \mu$. Now an application of a Clark's theorem (see [11, Theorem 9.1]) to $-I$ yields that $I$ has at least $\mathcal{N}(\lambda)$ distinct pairs of critical points.

\section{The SUblineAr CASE}

In this section we always assume that $(g 5)$ holds. It follows that

$$
c_{1}|u|^{\alpha} \leq G(x, u) \leq c_{2}\left(|u|^{\alpha}+|u|^{\beta}\right) \quad \text { for all }(x, u) .
$$

We consider the functional defined on $E_{\alpha}$

$$
J(u)=J_{\lambda}(u):=\int_{\mathbb{R}^{3}} G(x, u)+\frac{1}{2}\left\|u^{-}\right\|^{2}-\frac{1}{2}\left\|u^{+}\right\|^{2} .
$$

Then $J \in \mathcal{C}^{1}\left(E_{\alpha}, \mathbb{R}\right)$ and critical points of $J$ are solutions of (S).

If $\lambda \leq \lambda_{0}, E^{+}=\{0\}$ and clearly $J$ has no nontrivial critical points. Therefore we fix $\lambda \in\left(\lambda_{0}, \lambda_{e}\right)$ from now on. Note that $J$ is unbounded from either above or below.

Since $\operatorname{dim}\left(E^{+} \oplus E^{0}\right)<\infty$ and $\beta<2$, it follows from (3.1) that

$\left(J_{1}\right)$ There is $R>0$ such that $\sup J\left(E^{+} \backslash B_{R}\right) \leq 0$;

$\left(J_{2}\right)$ There is $\rho>0$ such that $\mu:=\inf J\left(S_{\rho}\right)>0$.

Let $\left\{e_{n}: n \in \mathbb{N}\right\}$ be a normal orthogonal base of $E_{\alpha}^{-}, Y_{n}:=\operatorname{span}\left\{e_{1}, \cdots, e_{n}\right\}$ and $E_{n}:=E^{+} \oplus E^{0} \oplus Y_{n}$. Note that $\operatorname{dim} E_{n}<\infty$. Set $J_{n}:=\left.J\right|_{E_{n}}$, the restriction of $J$ on $E_{n}$. A sequence $\left(u_{n}\right) \subset E_{\alpha}, u_{n} \in E_{n}$, is called a $(\mathrm{PS})_{c}^{*}$ sequence if $J\left(u_{n}\right) \rightarrow c$ and $J_{n}^{\prime}\left(u_{n}\right) \rightarrow 0 . J$ is said to satisfy the $(\mathrm{PS})_{c}^{*}$ condition if any $(\mathrm{PS})_{c}^{*}$ sequence has a convergent subsequence.

Lemma 3.1. $J$ satisfies the $(\mathrm{PS})_{c}^{*}$ condition.

Proof. Let $u_{n} \in E_{n}$ be such that $J\left(u_{n}\right) \rightarrow c$ and $\varepsilon_{n}=\left\|J_{n}^{\prime}\left(u_{n}\right)\right\| \rightarrow 0$. It follows from $(g 5)$ that

$$
J\left(u_{n}\right)+\varepsilon_{n}\left\|u_{n}\right\|_{\alpha} \geq \int_{\mathbb{R}^{3}}\left(G\left(x, u_{n}\right)-\frac{1}{2} g\left(x, u_{n}\right) u_{n}\right) \geq c\left|u_{n}\right|_{\alpha}^{\alpha},
$$

hence

$$
\left|u_{n}\right|_{\alpha} \leq c\left(1+\|u\|_{\alpha}^{1 / \alpha}\right)
$$


By the Hölder inequality

$$
\begin{aligned}
\int_{\mathbb{R}^{3}}\left|g\left(x, u_{n}\right) u_{n}^{+}\right| & \leq c \int_{\mathbb{R}^{3}}\left(\left|u_{n}\right|^{\alpha-1}+\left|u_{n}\right|^{\beta-1}\right)\left|u_{n}^{+}\right| \\
& \leq c\left(\left|u_{n}\right|_{\alpha}^{\alpha-1}\left|u_{n}^{+}\right|_{\alpha}+\left|u_{n}\right|_{\beta}^{\beta-1}\left|u_{n}^{+}\right|_{\beta}\right)
\end{aligned}
$$

and so, since $\operatorname{dim} E^{+}<\infty$,

$$
\int_{\mathbb{R}^{3}}\left|g\left(x, u_{n}\right) u_{n}^{+}\right| \leq c\left(\left\|u_{n}\right\|_{\alpha}^{\alpha-1}+\left\|u_{n}\right\|_{\alpha}^{\beta-1}\right)\left\|u_{n}^{+}\right\| .
$$

Therefore,

$$
\begin{aligned}
\left\|u_{n}^{+}\right\|^{2} & =\int_{\mathbb{R}^{3}} g\left(x, u_{n}\right) u_{n}^{+}-I_{n}^{\prime}\left(u_{n}\right) u_{n}^{+} \\
& \leq c\left(1+\left\|u_{n}\right\|_{\alpha}^{\beta-1}\right)\left\|u_{n}^{+}\right\|
\end{aligned}
$$

or

$$
\left\|u_{n}^{+}\right\| \leq c\left(1+\left\|u_{n}\right\|_{\alpha}^{\beta-1}\right) .
$$

Moreover, since

$$
\begin{aligned}
\left\|u_{n}^{-}\right\|^{2} & =2 I\left(u_{n}\right)+\left\|u_{n}^{+}\right\|^{2}-2 \int_{\mathbb{R}^{3}} G\left(x, u_{n}\right) \\
& \leq c\left(1+\left\|u_{n}\right\|_{\alpha}^{2(\beta-1)}\right)
\end{aligned}
$$

one has

$$
\left\|u_{n}^{-}\right\| \leq c\left(1+\left\|u_{n}\right\|_{\alpha}^{\beta-1}\right) .
$$

Hence, in virtue of $(3.2)-(3.4),\left(u_{n}\right)$ is bounded. We can suppose $u_{n} \rightarrow u$ and $u_{n}^{0}+u_{n}^{+} \rightarrow u^{0}+u^{+}$.

Let $P_{n}: E_{\alpha} \rightarrow E_{n}$ denote the projection. Then

$$
\begin{aligned}
J_{n}^{\prime}\left(u_{n}\right)\left(u_{n}^{-}-P_{n} u^{-}\right) & =\int_{\mathbb{R}^{3}} g\left(x, u_{n}\right)\left(u_{n}^{-}-P_{n} u^{-}\right)+\left(u_{n}^{-}, u_{n}^{-}-P_{n} u^{-}\right) \\
& =\int_{\mathbb{R}^{3}} g\left(x, u_{n}\right)\left(u_{n}-u\right)+\left(u_{n}^{-}, u_{n}^{-}-u^{-}\right)+o(1)
\end{aligned}
$$

or

$$
\int_{\mathbb{R}^{3}} g\left(x, u_{n}\right) u_{n}=\int_{\mathbb{R}^{3}} g\left(x, u_{n}\right) u-\left\|u_{n}^{-}\right\|^{2}+\left(u_{n}^{-}, u^{-}\right)+o(1) .
$$

Thus

$$
\limsup _{n \rightarrow \infty} \int_{\mathbb{R}^{3}} g\left(x, u_{n}\right) u_{n} \leq \int_{\mathbb{R}^{3}} g(x, u) u .
$$

On the other hand, by a Fatou lemma,

$$
\liminf _{n \rightarrow \infty} \int_{\mathbb{R}^{3}} g\left(x, u_{n}\right) u_{n} \geq \int_{\mathbb{R}^{3}} g(x, u) u .
$$

Thus

$$
\int_{\mathbb{R}^{3}} g\left(x, u_{n}\right) u_{n} \rightarrow \int_{\mathbb{R}^{3}} g(x, u) u .
$$

We obtain $\left\|u_{n}^{-}\right\| \rightarrow\left\|u^{-}\right\|$, which implies $u_{n}^{-} \rightarrow u^{-}$.

Similar to the above arguments one can prove the following lemma. 
Lemma 3.2. $J$ and $J_{n}$ satisfy the $(\mathrm{PS})_{c}$ condition.

Proof of Theorem 2. It follows from $\left(J_{1}\right)$ and $\left(J_{2}\right)$ that $J$ has the Mountain-Pass geometry [11, [15]. Since $J$ satisfies the $(\mathrm{PS})_{c}$ condition for all $c, J$ possesses a critical value $c \geq \mu$, hence conclusion $(i)$ follows.

Next we prove conclusion $(i i)$. We write gen $(A) \in \mathbb{N} \cup\{0, \infty\}$ for the Krasnoselski genus of a symmetric subset $A$ of $E_{\alpha}$, that is, $\operatorname{gen}(A)$ is the least integer $k$ such that there is an odd continuous map $A \rightarrow S^{k-1}$. If no such map exists, then $\operatorname{gen}(A):=\infty$. Let $n^{0}:=\operatorname{dim} E^{0}$ and set

$$
\begin{aligned}
\Gamma_{k}^{n}:=\left\{A \subset E_{n} \backslash\{0\}:\right. & \left.A \text { closed, symmetric, } \operatorname{gen}(A) \geq n+n^{0}+k\right\}, \\
& c_{k}^{n}:=\sup _{A \in \Gamma_{k}^{n}} \inf _{u \in A} J(u) .
\end{aligned}
$$

If $A \in \Gamma_{k}^{n}$, then $A \cap E^{+} \neq \emptyset$ (see [1]). Hence

$$
c_{k}^{n} \leq J\left(E^{+}\right)<\infty .
$$

Since gen $\left(S_{\rho} \cap E_{n}\right)=n+n^{0}+\mathcal{N}(\lambda)$, one has $S_{\rho} \cap E_{n} \in \Gamma_{k}^{n}$ for $k=1, \cdots, \mathcal{N}(\lambda)$, and consequently by $\left(J_{2}\right)$

$$
c_{k}^{n} \geq \inf J\left(S_{\rho} \cap E_{n}\right) \geq \mu .
$$

Since $J_{n}$ satisfies the $(\mathrm{PS})_{c}$ condition, $c_{k}^{n}$ is a critical value of $J_{n}$ for $k=1, \cdots, \mathcal{N}(\lambda)$. Clearly $\Gamma_{k+1}^{n} \subset \Gamma_{k}^{n}$ and one has

$$
c_{k}^{n} \geq c_{k+1}^{n} .
$$

Therefore, letting $c_{k}^{n} \rightarrow c_{k}$ as $n \rightarrow \infty$ (along a subsequence), we have

$$
c_{\mathcal{N}} \leq \cdots \leq c_{1}
$$

and, by $(\mathrm{PS})_{c}^{*}, c_{k}$ is a critical value of $J$.

It remains to show that if $c=c_{k}=\cdots=c_{k+j}$, then $\operatorname{gen}(\mathcal{K}(c)) \geq j+1$ where $\mathcal{K}(c):=\left\{u \in E_{\alpha}: J(u)=c, J^{\prime}(u)=0\right\}$. For this goal we note that, since $J(0)=0$ while $c \geq \mu>0, \quad 0 \notin \mathcal{K}(c)$. The continuity of genus implies that there is $\delta>0$ such that $\operatorname{gen}\left(N_{3 \delta}(\mathcal{K}(c))\right)=\operatorname{gen}(\mathcal{K}(c))$. Invoking $(\mathrm{PS})_{c}$ we have $\bar{\varepsilon}, \sigma>0$ and $\bar{n} \in \mathbb{N}$ satisfying

$$
\left\|J_{n}^{\prime}(u)\right\| \geq \sigma \quad \text { for all } u \in\left(J_{n}\right)_{c-\bar{\varepsilon}}^{c+\bar{\varepsilon}} \backslash N_{\delta}^{n}, n \geq \bar{n},
$$

where $N_{\delta}^{n}=N_{\delta}(\mathcal{K}(c)) \cap E_{n}$. By a standard deformation argument we choose $\varepsilon>0$ and construct a sequence of odd homeomorphisms $h_{n}: E_{n} \rightarrow E_{n}$ which maps $\left(J_{n}\right)_{c-\varepsilon} \backslash N_{\delta}^{n}$ into $\left(J_{n}\right)_{c+\varepsilon}$ (see [15]). Now choose a $n \geq \bar{n}$ large so that

$$
c-\varepsilon / 2<c_{k+j}^{n} \leq \cdots \leq c_{k}^{n}<c+\varepsilon / 2 .
$$

Choose $A \in \Gamma_{k+j}^{n}$ with

$$
\inf J(A)>c-\varepsilon / 2 \text {. }
$$

If $\operatorname{gen}(\mathcal{K}(c)) \leq j$, then

$$
\operatorname{gen}\left(\overline{A \backslash N_{\delta}^{n}}\right) \geq n+n^{0}+k+j-\operatorname{gen}(\mathcal{K}(c)) \geq n+n^{0}+k,
$$

that is, $\overline{A \backslash N_{\delta}^{n}} \in \Gamma_{k}^{n}$. Therefore $h_{n}\left(\overline{A \backslash N_{\delta}^{n}}\right) \in \Gamma_{k}^{n}$ and, consequently,

$$
c+\varepsilon \leq \inf J\left(h_{n}\left(\overline{A \backslash N_{\delta}^{n}}\right)\right) \leq c_{k}^{n} \leq c+\varepsilon / 2,
$$

a contradiction. 
Remark 3.3. There is a three-body system with two-body potentials, $V=V_{01}(x)+$ $V_{02}(y)+V_{12}(x-y)$, so that the number of bound states $E(\nu)$ of $A_{\nu}:=-\Delta_{x}-\Delta_{y}+\nu V$ has the following property: There is an increasing sequence $0<\nu_{1}<\nu_{2}<\ldots$ so that $E(\nu)=\infty$ if $\nu \in\left[\nu_{1}, \nu_{2}\right] \cup\left[\nu_{3}, \nu_{4}\right] \cup \cdots$ and $E(\nu)<\infty$ if $\nu \in \mathcal{T}:=$ $\left[0, \nu_{1}\right) \cup\left(\nu_{2}, \nu_{3}\right) \cup \cdots$; see 13 . All the previous existence and multiplicity results can be established for the equation $\left(\mathrm{S}_{\nu}\right)$ obtained from $(\mathrm{S})$ with $A$ replaced by $A_{\nu}$ in parallel.

Theorem 3.4. Let $\nu \in \mathcal{T}$. (a) Assume $(g 1)-(g 3)$. Then $\left(\mathrm{S}_{\nu}\right)$ has no nontrivial solutions if $\lambda \leq \lambda_{0}$, has at least two nontrivial solutions if $\lambda \in\left(\lambda_{0}, \lambda_{e}\right)$, and has $\mathcal{N}(\lambda)$ pairs solutions if $(g 4)$ also holds. (b) Assume $(g 5)$ holds. Then $\left(\mathrm{S}_{\nu}\right)$ has no nontrivial solutions if $\lambda \leq \lambda_{0}$, has at least one nontrivial solution if $\lambda \in\left(\lambda_{0}, \lambda_{e}\right)$, and has $\mathcal{N}(\lambda)$ pairs solutions if $(g 4)$ also holds.

\section{REFERENCES}

[1] S. Alama and Y. Y. Li, On "Multibump" bound states for certain semilinear elliptic equations, Indiana J. Math. 44 (1992), 983-1026. MR 94d:35044

[2] S. Alama and G. Tarantello, On the solvability of a semilinear elliptic equation via an associated eigenvalue problem, Math. Z. 221 (1996), 467-493. MR 97d:35067

[3] A. Ambrosetti, M. Badiale and S. Cingolani, Semiclassical states of nonlinear Schrödinger equations, Arch. Rat. Mech. Anal. 140 (1997), 285-300. MR 98k:35172

[4] T. Bartsch and Y. H. Ding, On a nonlinear Schrödinger equation with periodic potential, Math. Ann. 313 (1999), 15-37. MR 99m:35218

[5] R. Benguria, H. Brézis and E. H. Lieb, The Thomas-Fermi-von Weizäcker theory of atoms and molecules, Comm. Math. Phys. 79 (1981), 167-180. MR 83m:81114

[6] V. Coti-Zelati and P. H. Rabinowitz, Homoclinic type solutions for a semilinear elliptic PDE on $\mathbb{R}^{N}$, Comm. Pure Appl. Math. 45 (1992), 1217-1269. MR 93k:35087

[7] M. A. Del Pino and P. Felmer, Multiple solutions for a semilinear elliptic equation, Trans. Amer. Math. Soc. 347 (1995), 4839-4853. MR 96c:35062

[8] W. Kryszewski and A. Szulkin, Generalized linking theorem with an application to semilinear Schrödinger equation, Adv. Diff. Eq. 3 (1998), 441-472. CMP 2000:11

[9] S.J. Li and M. Willem, Applications of local linking to critical point theory, J. Math. Anal. Appl. 189 (1995), 6-32. MR 96a:58045

[10] P. L. Lions, Solutions of Hartree-Fock equations for Coulomb systems, Comm. Math. Phys. 109 (1987), 33-97. MR 88e:35170

[11] P.H. Rabinowitz, Minimax Methods in Critical Point Theory with Applications to Differential Equations, C. B. M. S. 65, Amer. Math. Soc., Providence, R. I., 1986. MR 87j:58024

[12] P. H. Rabinowitz, On a class of nonlinear Schrödinger equations, ZAMP 43 (1992), 270-291. MR 93h:35194

[13] M. Reed and B. Simon, Methods of Mathematical Physics, vol. I-IV, Academic Press, 1978. MR 58:12429a MR 58:12429b MR 80m:81085 MR 58:12429c

[14] C. Troestler and M. Willem, Nontrivial solution of a semilinear Schrödinger equation, Comm. Partial Diff. Eq. 21 (1996), 1431-1449. MR 98i:35034

[15] M. Willem, Minimax Methods, Birkhäuser, Boston, 1996.

Morningside Center of Mathematics and institute of Mathematics, AMSS, Chinese Academy of Sciences, 100080 Beijing, People's Republic of China

E-mail address: dingyh@math03.math.ac.cn 